\title{
AUDIT
}

\section{An audit on ventilator management in critical care units in National Hospital Sri Lanka}

\author{
B K Udya Nirmalle Rodrigo \\ Registrar in Anaesthesiology, National Hospital of Sri Lanka
}

*Corresponding author : bkun1977@yahoo.co.uk

\begin{abstract}
Mechanical ventilation is a common life saving intervention in Intensive Care Units (ICU). Traditional approaches to mechanical ventilation use large tidal volumes which may cause volutrauma. Lung protective ventilator strategies are used to prevent this. Concept of a ventilator care bundle was introduced to improve the quality of care in ventilated patients.
\end{abstract}

The main objectives of this audit were to find out the common modes and ventilator parameters used in our ICUs and whether ventilator care bundle are used. The audit was conducted in Feb 2011. Data was collected from intubated and mechanically ventilated patients in 5 critical care units at National Hospital of Sri Lanka (NHSL). Total number of patients analyzed was 100. Ethical clearance was obtained from the NHSL ethics committee.

Of the 100 patients 65 were males and 35 were females. Highest number of patients belonged to age group of 51-60yrs, and the majority were from Neuro-Trauma ICUs (NT-ICU). Commonest mode of ventilation was SIMV/PC (45\%) and CMV/VC(37\%). Frequently used tidal volume/kg was $7.1-10 \mathrm{ml} / \mathrm{kg}(63 \%)$ and the lung protective tidal volume $/ \mathrm{kg}$ range of $4.1-7 \mathrm{ml} / \mathrm{kg}$ was used only in $31 \%$. Commonest reasons for ventilation were central nervous system (CNS) (32\%) and respiratory causes $(16 \%)$. Muscle relaxants were used in $54 \%$ of patients, mainly in NT-ICUs and AS-ICU. Reasons for muscle relaxation were CNS stabilization following head injury/ neurosurgical intervention $(28 \%)$, and to control intra-cranial pressure $(11 \%) .91 \%$ of patients were given sedation and sedation free window was practiced in $70 \%$ of patients. Head-up tilt was maintained in $89 \%$ of patients and the reasons for not maintaining that position was severe hypotension and cervical spine injury. Mouth care (100\%) and antacid prophylaxis (99\%) were practiced in almost all the patients. Regular ETT cuff pressure checking is not a common practice in our ICUs.

This audit highlight that less than $1 / 3$ of ICU personnel practice lung protective ventilation strategies. To improve the quality of care of ventilated patients, we recommend to conduct awareness programmes to educate the ICU staff on the importance of practicing lung protective ventilator strategies and to introduce a system for monitoring compliance to the care bundle.

Keywords : Mechanical ventilation, ventilator care bundle

\section{Background}

Mechanical ventilation is a common and life saving intervention done in an ICU. Traditional approach to mechanical ventilation using large tidal volumes may cause volutrauma. The use of lung protective ventilator strategies hopes to prevent this. In view of preventing complications of mechanical ventilation the concept of a ventilator care bundle has been introduced worldwide.

Critically ill patients affected by severe acute respiratory failure need mechanical ventilation to survive. Mechanical support buys time for the lungs to heal. Nevertheless, $40-50 \%$ still die. ${ }^{1}$ Several studies suggest that mechanical breathing can also cause lung damage and bleeding. A new lung-protective way of mechanical ventilation was tested in large trials. These revealed that lung protective ventilation can decrease death in the short term, but the effects in the long term are uncertain or unknown. ${ }^{1,2,3}$

Evidence from ARDSNet trial revealed that ventilation strategy using a tidal volume equal or 
less than $7 \mathrm{ml} / \mathrm{kg}$ of measured body weight and plateau pressure less than $31 \mathrm{~mm} \mathrm{H}_{2} \mathrm{O}$ reduced mortality at day $28 .{ }^{1}$

Between 5-15\% of hospital patients develop infection during admission to ICU. ICU patients are 5-10 times more likely to acquire nosocomial $D$ infections than patients in the wards and approximately $86 \%$ of hospital associated pneumonia is linked with mechanical ventilation. Approximately $10-28 \%$ of critical care patients develop ventilator associated pneumonia. (VAP). VAP is also the most common and fatal infection of ICU and in the United States it affects 9-27\% of intubated patients and doubles the risk of mortality as compared with similar patients without VAP. VAP may account for up to $60 \%$ of all healthcare associated infections. VAP increases length of ICU stay by $28 \%$ and each incidence of VAP is estimated to generate an increased cost of $\$ 6000$ $\$ 22000 .^{2}$

No randomized controlled trials of a care bundle intervention were identified. The only identified studies of care bundles were two studies that recorded VAP rates before and after the introduction of a 'ventilator bundle'. Both studies used the Institute of Health care Improvement (IHI) care bundle as a basis, but added other components to it. A study reported in 2005 that ICUs with the highest rates of compliance with the ventilator bundle had the highest rates of VAP reduction. With $>95 \%$ compliance, VAP rates decreased from 6.6 to 2.7 per 1,000 ventilator days, giving a 59\% reduction. Another study reported that a decrease in VAP rate occurred when compliance with the care bundle was audited and feedback given to the staff on both daily and weekly basis, but it was noted that compliance was not always $100 \%$, and varied from week to week. It was also noted that not every patient would be able to meet the criteria for all the care bundle components. $^{4}$

There is a scarcity of published research on the use of care bundles for the prevention of VAP. The choice of components of a care bundle would be based on a literature review and the unit clinicians' experience-based recommendations on which component to include and those which are medically contraindicated. ${ }^{4}$

According to the article by the IHI a care bundle is, in general, a grouping of best practices with respect to a disease process that individually improve care, but when applied together result in substantially greater improvement.

The key components of the IHI Ventilator Bundle are: $^{6}$

Elevation of the head of the bed

Daily "Sedation Vacations" and assessment of

readiness to extubate

Peptic Ulcer Disease (PUD) prophylaxis

Deep Venous Thrombosis (DVT) prophylaxis

Daily oral care with Chlorhexidine

Whilst each individual component of a care bundle will have an effect, it is not possible to attribute what effect each component has. For the results to be generalisable, all components need to be implemented and considered as a whole. ${ }^{4}$

So, the main objectives of this audit were to find out the commonly used ventilator modes and ventilator parameters in our Intensive Care Units (ICU) and the use of certain components of the ventilator care bundle in our ICUs.

\section{Material and method:}

The audit was carried out from $1^{\text {st }}$ to $28^{\text {th }}$ February 2011, in 5 different critical care units at National Hospital of Sri Lanka (NHSL). These ICUs included Medical (MICU), Surgical (SICU), Neuro-trauma and Neurosurgical (NT/NS ICUs), Accident Service (AS-ICU) and Cardio-thoracic (CT ICU). Data was collected from intubated and mechanically ventilated patients in the above ICUs.

\section{Ethical considerations:}

Confidentiality was strictly maintained. The care of the patient was not affected by the audit.

\section{Audit setting and the population:}

There were altogether 64 beds (SICU-8, MICU-8, NT/NS ICU-36, AS-ICU-6 and CT-ICU-6) in the 5 ICUs. NT/NS ICUs were the largest group which comprised of 6 ICUs including ETU. Admission policy and the type of patients were different from one another as each of these were specialized ICUs. Total study population comprised 100 patients.

\section{Data collection and the method:}

Data were obtained through data collection forms which were filled by a postgraduate trainee in anaesthesiology and critical care, appointed to the relevant ICU during that particular period. Patients 
records such as bed head ticket (BHT), monitoring charts maintained by critical care nurses and direct questioning of the critical care nurses were the added sources of data.

\section{Analysis of the data:}

Following areas were addressed during the analysis of the data.

1. Ventilator strategies were assessed as the common modes of ventilation and the tidal volume per mass body weight.

2. The components of ventilator care bundle assessed in this audit were;

- $\quad$ Practice of sedation hold window, head up position, mouth care and endotracheal tube cuff pressure measurement.

- Use of gastric ulcer prophylaxis

- The use of muscle relaxants in different ICUs.

\section{Results :}

During the period of audit total number of patients analyzed was 100, of which 65 were males. Their distribution among the different ICUs is shown in Table 1.

Table 1. Distribution of patients according to the gender \& ICU

\begin{tabular}{|l|c|c|c|}
\hline \multicolumn{1}{|c|}{ ICU } & Male & Female & Total \\
\hline NT/NS ICU & 21 & 10 & 31 \\
MICU & 14 & 15 & 29 \\
SICU & 11 & 05 & 16 \\
ASICU & 11 & 02 & 13 \\
CT ICU & 08 & 03 & 11 \\
Total & $\mathbf{6 5}$ & $\mathbf{3 5}$ & $\mathbf{1 0 0}$ \\
\hline
\end{tabular}

Table 2. Age distribution

\begin{tabular}{|l|c|}
\hline Age Group (years) & No. \\
\hline$<10$ & 3 \\
$11-20$ & 7 \\
$21-30$ & 9 \\
$31-40$ & 16 \\
$41-50$ & 15 \\
$51-60$ & 23 \\
$61-70$ & 17 \\
$>71$ & 10 \\
Total & $\mathbf{1 0 0}$ \\
\hline
\end{tabular}

Figure 1. Mode of ventilation (on Day 1)

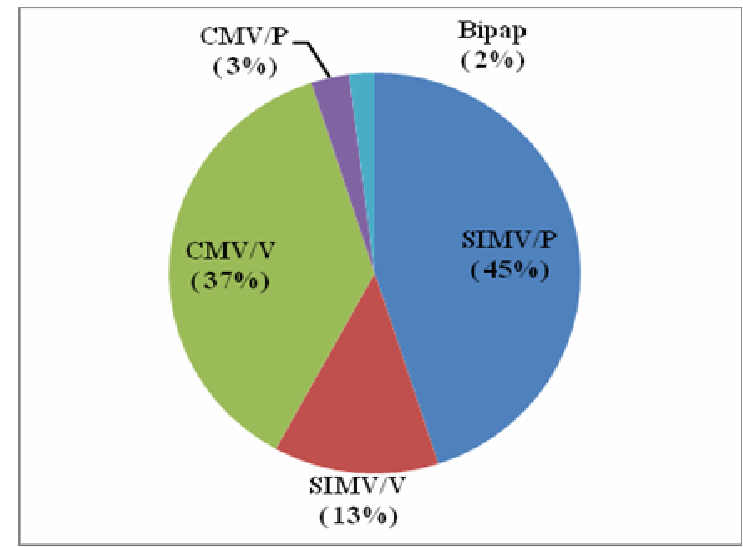

Figure 2. Tidal Volume/kg

Tidal volume was calculated obtaining the average value of 5 consecutive tidal volume values (using monitoring charts) on Day 1 after commencement of invasive ventilation.

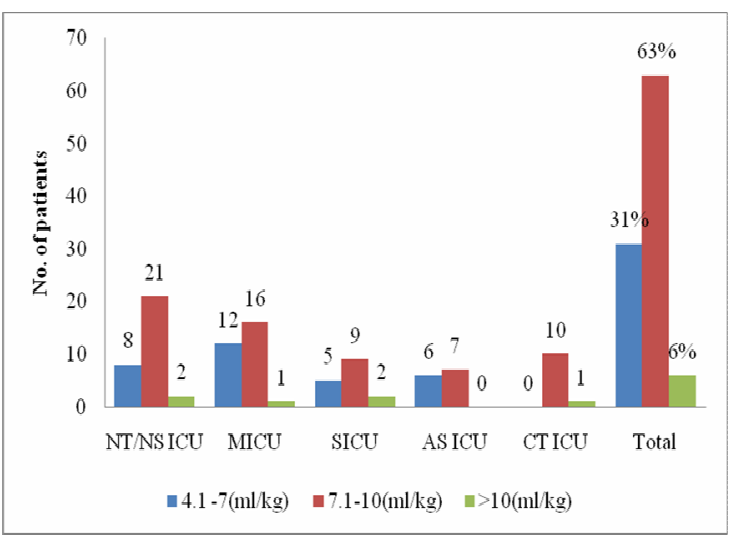

Figure 3. Sedation free window practice

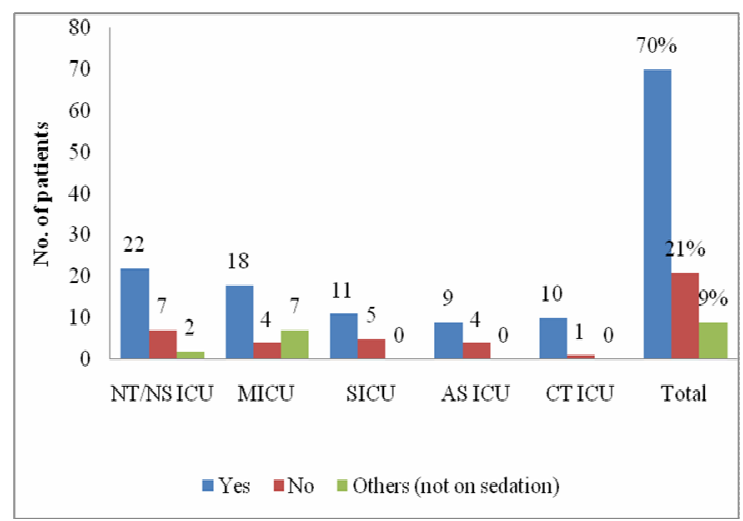


Table 3. Use of sedative drugs

\begin{tabular}{|l|l|}
\hline Drug & No. \\
\hline IV Midazolam & 42 \\
IV Midazolam + IV Morphine & 11 \\
IV Midazolam + IV Fentanyl & 03 \\
IV Midazolam + IV Propofol & 02 \\
IV Morphine + IV Propofol & 09 \\
IV Morphine & 20 \\
IV Propofol & 02 \\
IV Fentanyl & 02 \\
None & 09 \\
Total & $\mathbf{1 0 0}$ \\
\hline
\end{tabular}

Mouth care and head-up position

All the patients in this study population (100\%) had received mouth care and $89 \%$ were kept in propped-up position during the period of invasive ventilation.

Table 4. Use of antiseptic agents

\begin{tabular}{|l|l|}
\hline Antiseptic agent & No. \\
\hline Lime & 77 \\
Salt & 35 \\
Betadine & 40 \\
Normal saline & 4 \\
Toothpaste & 20 \\
$\mathrm{NaHCO}_{3}$ & 2 \\
Chlorhexidine $_{\text {Nystatin }}$ & 2 \\
\hline
\end{tabular}

Table 5. ETT cuff pressure checking

\begin{tabular}{|l|l|l|}
\hline ETT cuff pressure checking & yes & No \\
\hline NT/NS ICU & 1 & 30 \\
MICU & 5 & 24 \\
SICU & 16 & 0 \\
ASICU & 0 & 13 \\
CT ICU & 5 & 6 \\
Total (100) & $\mathbf{2 7}$ & $\mathbf{7 3}$ \\
\hline
\end{tabular}

\section{Gastric acid suppression}

In this population $99 \%$ had received gastric acid suppression.
Table 6. Use of muscle paralysis

\begin{tabular}{|l|c|c|}
\hline ICU & Yes & No \\
\hline NT/NS ICU & 27 & 4 \\
MICU & 6 & 23 \\
SICU & 7 & 9 \\
AS ICU & 13 & 0 \\
CT ICU & 1 & 10 \\
Total (100) & $\mathbf{5 4}$ & $\mathbf{4 6}$ \\
\hline
\end{tabular}

Discussion: In the study population of 100 patients 65 were males, with the highest number of patients belonging to the age group of 51-60yrs, followed by the 61-70yr group. Lowest number of patients was in the age group of 11-20yrs. It could be due to the lack of paediatric ICU representation in the audit sample. The majority represented from Neuro-trauma ICUs (which comprised of six ICUs).

The commonest mode of ventilation used was synchronised intermittent mandatory ventilation in pressure control mode (SIMV/PC) (45\%) and then controlled mandatory ventilation (CMV) (37\%). Only few patients were ventilated using BiPAP mode of ventilation (2\%). This could be due to unavailability of that mode of ventilation in most of the ventilators used in our ICUs. None of the patients in the sample were ventilated using Bilevel mode of ventilation.

Frequently used tidal volume range was 7.1$10 \mathrm{ml} / \mathrm{kg}$, which was used in $63 \%$ and then $4.1-$ $7 \mathrm{ml} / \mathrm{kg}$ in $31 \%$. Less than $1 / 3$ of the patients in this sample were ventilated using lung protective tidal volumes.

Muscle relaxants were used in $54 \%$ of patients, mainly in NT/NS-ICUs and ASICU. The reasons for use of muscle relaxants was CNS stabilization following head injury / neurosurgical intervention (28\%), and to stabilize the patient without fighting the invasive ventilation (11\%).

91\% of patients were given sedation and 9\% patients were not on sedation. The commonly used sedative agents were i.v. midazolam (42\%) and i.v. morphine $(20 \%)$. A combination of both was used in $11 \%$. Sedation free window was practiced in $70 \%$ of patients. The main reason for not practicing was when the patient was paralysed for ventilation. Head-up tilt was maintained in $89 \%$ of patients and the reasons for not maintaining that position were severe hypotension and cervical 
spine injury. Use of mouth care practice was highly efficient (100\%) and commonly used agents for mouth care were lime, salt and betadine. Chlorhexidine was used only in CT-ICU. Antacid prophylaxis (99\%) was practiced in almost all the patients and it was due to negligence that only one patient in the sample was not given gastric acid suppression. Only $27 \%$ of patients were subjected to ETT cuff pressure checking highlighting that ETT cuff pressure checking is not a common practice in our critical care units.

The use of DVT prophylaxis in our critical care units has not been assessed in this audit, in order to conduct a detailed assessment in a separate audit and also to make this audit a simpler one.

Treatment with a ventilation approach designed to protect the lungs from excessive stretch resulted in improvements in several important clinical outcomes in patients with acute lung injury and acute respiratory distress syndrome. On the basis of these results, high priority should be given to prevent excessive stretch of lung during adjustments to mechanical ventilation, and this lower-tidal-volume protocol should be used in patients with acute lung injury and the acute respiratory distress syndrome. ${ }^{1}$

The introduction of ventilator care bundles has been shown to reduce both ventilator days and length of stay in intensive care. Every ICU should have guidelines for the application of care bundles to mechanically ventilated patients. ${ }^{2}$

Gastrointestinal haemorrhage occurs in $9 \%$ of ventilated patients, with clinically important bleeding in $2 \%$. Prophylaxis with drugs or enteral feeding reduces this incidence. ${ }^{3}$

The supine position is a factor in the development of ventilator associated pneumonia. ${ }^{4}$ The semirecumbent position (45 degrees head up) reduces the incidence of clinically suspected or microbiologically proven pneumonias (relative risk reduction of $26 \%$ and $18 \%$ respectively).Continuous intravenous sedation may prolong the duration of mechanical ventilation and length of stay in the ICU. A daily sedation hold has been shown to reduce the duration of mechanical ventilation by more than 2 days, and total ICU stay by 3.5 days. ${ }^{5}$

It has been shown that excessive cuff pressures can cause ischaemia and necrosis of the tracheal mucosa despite the introduction of large volume, low pressure cuffs. Also, large volume cuffs can exert a high pressure on the tracheal wall if inflated past their resting volume. Excessive pressures must be prevented in all patients by objective measurement. When the cuff pressure required to prevent a leak is high, options include changing the size or type of tube, changing the mode of ventilation to reduce peak airway pressure, placing a tracheostomy below the area of dilation or accepting a small leak. ${ }^{5}$

\section{Conclusion:}

This audit highlights that less than $1 / 3$ of the population (31\%) was subjected to lung protecting ventilation strategies and our practice depends on the reason for ventilation. The practice of ventilator care bundle components such as sedation hold, maintenance of head-up position, antacid prophylaxis and practice of mouth care were towards the recommended standard of practice on ventilator care bundle. The common reasons for ignorance are absence of a clear written guideline relating to the care bundle, ignorance among staff of local guidelines and inadequate system for monitoring compliance.

\section{Recommendation:}

To achieve the best practice in our critical care units it is advisable to maintain a Ventilator Bundle checklist (tick box type fact sheet) such as provided below to assess the compliance of each ICU with implementing each element of the ventilator care bundle. The greater the level of compliance with all items in the bundle, the better the reduction in ventilator-associated pneumonia rate.

\section{Ventilator Bundle checklist.}

\begin{tabular}{|l|l|l|l|l|l|}
\hline Patient :.................... & & & & & \\
\hline Date of admission : ......... & & & & & \\
\hline \multicolumn{1}{|c|}{ ICU Day } & $\mathbf{1}$ & $\mathbf{2}$ & $\mathbf{3}$ & $\mathbf{4}$ & $\mathbf{5}$ \\
\hline Head elevation & & & & & \\
\hline $\begin{array}{l}\text { Sedation hold and assess } \\
\text { readiness to extubation }\end{array}$ & & & & & \\
\hline PUD prophylaxis & & & & & \\
\hline DVT prophylaxis & & & & & \\
\hline Mouth care & & & & & \\
\hline
\end{tabular}

(Adapted from: Dominican Hospital, Santa Cruz, California, USA)

Frequent audits should be conducted in each unit to find out the incidence of omissions of individual elements of the care bundle, deviations from policy and the reasons for this. Re-auditing is also 
equally important which gives an idea about how far the implementations are being carried-out. Team discussions, workshops, seminars and awareness programmes for all categories of ICU staff should be organized time to time towards updating the knowledge.

\section{References:}

1. ARDS network: The New England Journal of Medicine.2000;342:1301-1308

http://dx.doi.org/10.1056/NEJM200005043421801

PMid:10793162

2. Rello, J., Ollendorf, D. A., Oster, G., Vera-Llonch, M., Bellm, L., Redman, R., Kollef, M. H., and VAP Outcomes Scientific Advisory Group. Epidemiology and outcomes of ventilator-associated pneumonia in a large US database. Chest 2002; 122: 2115-2121. http://dx.doi.org/10.1378/chest.122.6.2115

PMid:12475855

3. Cook DJ et al. Incidence of clinically important bleeding in mechanically ventilated patients. J Intens CareMed1991;6:167-174.

4. Kollef M. The prevention of ventilator associated pneumonia. NEngJMed.1999;340:627-634. http://dx.doi.org/10.1056/NEJM199902253400807

PMid:10029648

5. SICSAG (Scottish Intensive care audit group) VAP Prevention Bundle, Guidance for Implementation document. www.sicsag.scot.nhs.uk

6. Institute for Health Care Improvement; Ventilator Bundle. www.ihi.org/ventilator bundle

\section{THE COLLEGE OF ANAESTHESIOLOGISTS OF SRI LANKA}

\section{LECTURE DEMONSTRATION \& CERTIFICATION}

ON

\section{Cardio Pulmonary Resuscitation}

$\begin{array}{ccc}\text { Venue } & - & \text { Trauma Lecture Hall } \\ \text { Date } & - & \text { National Hospital, Sri Lanka } \\ \text { Time } & - & \text { on Tuesdays } \\ & & 9.00 \text { am } \\ & \text { Conducted by } \\ & \text { Dr Manjula Kularatne } \\ \text { Consultant Anaesthetist, NSU } \\ \text { Contact } \\ \text { Office: } 2672327\end{array}$

$* * * * * * * * * * * * * * * * * * * * * * * * * * * * * * * * * * * * * * * * * * * * * * * * * * * * * * * * * * * *$ 